\title{
RELACIÓN ENTRE METODOLOGÍAS DOCENTES Y ENFOQUES DE APRENDIZAJE EN LA UNIVERSIDAD
}

\author{
Marta García Jiménez \\ Facultad Ciencias de la Educación \\ Universidad de Granada \\ martagj@ugr.es \\ María Fernández Cabezas \\ Facultad Ciencias de la Educación \\ Universidad de Granada
}

Recepción Artículo: 14 Febrero 2020

Admisión Evaluación: 4 marzo 2020

Informe Evaluador 1: 18 marzo 2020

Informe Evaluador 2: 20 Marzo 2020

Aprobación Publicación: 20 abril 2020

\section{RESUMEN}

El Espacio Europeo de Educación Superior (EEES) requiere un giro en la percepción del proceso de enseñanza-aprendizaje. El objetivo principal de este estudio es conocer las correlaciones existentes entre las metodologías docentes Ilevadas a cabo y su relación con los enfoques y estrategias de aprendizaje utilizadas por el alumnado de primer y de tercer curso del grado en Pedagogía de la Facultad de Ciencias de la Educación de la Universidad de Granada. Los instrumentos de recogida de información han sido el Revised two- Factor Study Process Questionnaire (R-SPQ- 2F), el Inventario de hábitos de estudio (I.H.E) y el Cuestionario de Evaluación de las Variables "Moduladoras" del Estilo de Enseñanza en Educación Superior (CEMEDEPU). Para el análisis de datos se han realizado estadísticos descriptivos, una comparación de medias mediante el análisis de T de Student y se han analizado las correlaciones entre variables de los cuestionarios I.H.E. y R-SPQ-2F. Los resultados señalan que la metodología docente centrada en el aprendizaje influye directamente en el enfoque profundo, la motivación profunda y la estrategia profunda. Se obtiene una relación directa y significativa del enfoque profundo con las escalas de condiciones ambientales del estudio, planificación del estudio y asimilación de contenidos.

Palabras clave: educación superior; estrategias de aprendizaje; modelos de enseñanza; enfoques de aprendizaje

\section{ABSTRACT}

The main objective of this study is to know the correlations between the teaching methodologies carried out and their relationship with the learning approaches and learning strategies used by students of first and third course of the Grade on Pedagogy of the Faculty of Science Education of the University of Granada. The instru- 


\section{RELACIÓN ENTRE METODOLOGÍAS DOCENTES Y ENFOQUES DE APRENDIZAJE EN LA UNIVERSIDAD}

ments for collecting information have been the Revised Two-Factor Study Process Questionnaire (R-SPQ-2F), the Inventory of study habits (IHE) and the Questionnaire for the Evaluation of the "Modulating" Variables of the Teaching Style in Higher Education (CEMEDEPU). The data analysis has been of descriptive statistics, a comparison of means by Student's T analysis and the correlations between variables of the I.H.E. and R-SPQ-2F have been analyzed. The results indicate that the teaching methodology focused on learning directly influences the deep focus, deep motivation and deep strategy. Is obtained a direct and significant relationship of the deep approach with the scales of environmental conditions of the study, study planning and content assimilation is obtained.

Keywords: higher education; learning strategies; teaching models; approaches to learning

\section{ANTECEDENTES}

La universidad en el EEES requiere cambios a nivel: estructural, implementando un sistema de créditos 'ECTS', reconocerían aquellas horas destinadas al aprendizaje autónomo y autorregulado; una reestructuración de las titulaciones de Grado y Posgrado para integrar al alumnado en el mercado laboral vigente; y organizativo, un modelo de aprendizaje centrado en el alumnado. Su finalidad es mejorar la calidad y excelencia de la educación universitaria (Cruz-Tomé, 2003; García-Berbén, 2008; Pascual, 2015).

Esta adaptación supone un cambio en el paradigma del proceso de enseñanza - aprendizaje (E-A) (Biggs, 1999; Prosser y Trigwell, 1999; Ramsden, 2003), conlleva una formación integral del alumnado desde una modificación en la mentalidad del profesorado hasta una nueva concepción del proceso (adquisición de competencias generales y específicas) (Cruz- Tomé, 2003; Pascual, 2015).

\section{Concepción de los procesos de enseñanza-aprendizaje}

La concepción de los procesos E-A en las universidades españolas han sufrido cambios abruptos para adaptarse al EEES, la función del profesorado se configura como 'guía' en el proceso de aprendizaje, el alumnado adquiere un aprendizaje más autónomo y autorregulado para obtener sus metas académicas y adapta las estrategias de aprendizaje para lograrlas. (Cruz- Tomé, 2003; Fernández de Haro, 2017; Koivuniemi, Panadero, Malmberg y Järvelä, 2017; Pascual, 2015; Pintrich, 2000; Zimmerman, 2000).

En la actualidad se genera constantemente nueva información, que hay que manejar, comprender y asimilar, esto requiere cambios en los procesos de E-A que otorguen al alumnado la capacidad de seleccionar y adquirir información relevante de su ámbito (Valerio, Jaramillo, Caraza y Rodríguez, 2016).

\section{Concepción de la enseñanza. Teorías}

Desde la década de los '50 las concepciones de E-A han sufrido reformulaciones, empezó a tomar relevancia la perspectiva cognitiva en las áreas de Psicología y Educación (García-Berbén, 2008).

La concepción de Ramsden (2003) sobre la enseñanza converge en distintas teorías organizadas jerárquicamente sustentadas por el paradigma fenomenológico: Teoría de enseñanza como transmisión: el profesor es el foco del proceso de E-A y el alumnado sujeto pasivo; Teoría de enseñanza como organización de la actividad del alumnado: el profesor es un 'guía', adapta estrategias y métodos de enseñanza para fomentar y motivar el aprendizaje y el alumnado es sujeto activo; y Teoría de la enseñanza como facilitación del aprendizaje: la enseñanza se centra en la cooperación entre profesorado- alumnado, varía en función del contenido a adquirir y del alumnado. Vermunt (2003) señala que este tipo de enseñanza basada en solucionar problemas es la más idónea y eficaz en el contexto universitario.

\section{Concepción del aprendizaje. Metáforas de Mayer.}

Las distintas concepciones del aprendizaje dependen de la perspectiva estudiada, Mayer realizó un estudio comparativo sobre las perspectivas conductual y cognitiva. Esta última dividida en dos líneas de investigación, que dio lugar a tres metáforas: "(1) aprendizaje como adquisición de respuestas, (2) aprendizaje como adquisición de conocimiento y (3) aprendizaje como construcción del significado" (1992, citado en García-Berbén, 2008, p.11). 
La primera metáfora vinculada a una perspectiva conductual y las otras dos a una perspectiva cognitiva. Desde la perspectiva cognitiva: la segunda metáfora entiende al alumnado como receptor de información y el profesorado transmite contenidos curriculares; y la tercera metáfora es un método de enseñanza basado en la construcción del aprendizaje, el alumnado lo construye de forma activa otorgándole significado y autorregulándose, siendo relevante las estrategias cognitivas y metacognitivas utilizadas en cada etapa (García-Berbén, 2008).

\section{Enfoques de aprendizaje.}

Los primeros estudios sobre enfoques de aprendizaje fueron realizados por Marton (Marton, 1975; Marton y Säljö, 1976a, 1976b, 1984; Marton y Svensson, 1979) en Suiza. Marton y Säljö (1976a y b) analizaron cómo el alumnado universitario afrontaba la lectura de trabajos académicos. Las respuestas obtenidas se agrupaban en: 'procesamiento profundo' y 'procesamiento superficial', los resultados mostraron cómo percibían la tarea académica y su intención. Se interpretó que dependiendo de la intención daría lugar a un tipo de procesamiento, diferentes grados de comprensión. Por ello establecieron nuevos términos 'enfoque profundo' y 'enfoque superficial'. A partir de estos estudios surgen nuevos y añadieron un tercero 'enfoque de logro' (Biggs, 1988; Biggs, Kember y Leung, 2001; Marton y Säljö, 1984).

Biggs (1987) construyó el Learning Process Questionnaire (LPQ), para alumnado de Secundaria; y el Study Process Questionnaire (SPQ), para alumnado universitario. Ambos cuestionarios medían los tres enfoques y están agrupados en seis subescalas: estrategia y motivación (profunda; superficial; de logro). Diversas investigaciones demostraron que la naturaleza del enfoque de logro es distinta a la de los otros enfoques, su principal componente es la planificación y organización del tiempo y espacio, por tanto, implica la autoestima del alumnado (Biggs, 1987, 1988, 1993; Entwistle, 1988; Valle, Cabanach y Vieiro, 1997). Debido a esto, Biggs et al. (2001) presentaron una versión mejorada del SPQ (R-SPQ-2F).

Los enfoques de aprendizaje fueron definidos por Biggs (1989, 1993, 2001): el enfoque superficial, el alumnado tiene motivación extrínseca, realiza las tareas con mínimo esfuerzo y evita fracasar. Utiliza estrategias de memorización; el enfoque profundo, el alumnado se preocupa por aprender (motivación intrínseca) y utiliza estrategias de comprensión, relacionando contenidos y conocimientos entre sí y los vincula con previos.

Martínez y Galán (2000) enfatizan que los procesos de E-A orientados a la construcción de aprendizaje, requiere que el alumnado disponga de estrategias de aprendizaje que le permitan realizar las tareas con éxito, por lo que se han generado instrumentos para medir los procesos internos (cognitivos y motivacionales). Rinaudo, Chiecher y Donolo (2003) afirman que influye positivamente en el aprendizaje los elevados niveles de motivación intrínseca y el uso adecuado de estrategias de aprendizaje.

\section{Estrategias de aprendizaje. Concepto y tipologías.}

Las estrategias de aprendizaje se definen como procedimientos cognitivos internos que se utilizan para procesar, adquirir, almacenar y evocar información para posteriormente transferirla (Weinstein y Mayer, 1986).

Cano (2007) las divide en: estrategias cognitivas, son procedimientos de carácter intencional, le otorgan al alumnado la capacidad de tomar decisiones adecuadas para su sistema cognitivo. Se consideran estrategias cognitivas el repaso (procesamiento superficial de la información); organización, elaboración y pensamiento crítico (procesamiento profundo); estrategias metacognitivas controlan la comprensión de la información y regulación de los procesos mentales utilizados para alcanzar las metas académicas propuestas. Se consideran estrategias metacognitivas la planificación, la regulación y la evaluación; y estrategias de apoyo, el control que se ejerce en recursos de carácter no cognitivo y se utiliza para mejorar el rendimiento académico. Se consideran estrategias de apoyo la motivación, el afecto y las actitudes.

\section{Estudios recientes. Enfoques y estrategias de aprendizaje.}

El proceso educativo en el ámbito universitario (EEES) tiene como finalidad enseñar al alumnado a aprender 


\section{RELACIÓN ENTRE METODOLOGÍAS DOCENTES Y ENFOQUES DE APRENDIZAJE EN LA UNIVERSIDAD}

mediante la utilización de modelos activos de enseñanza que requieren un uso adecuado de estrategias de aprendizaje (Romero, Jordan, Ilaquiche, García y Tapia, 2019).

Un estudio de Alarcón, Alcas, Alarcón, Natividad y Rodríguez (2019) vinculado a las estrategias de aprendizaje usadas en educación superior en Perú, señala que para alcanzar un aprendizaje autónomo y autorregulado se requiere un buen uso de ellas.

González-García, Sánchez- García, Nieto- Librero y Galindo- Villardón (2019) relaciona la actitud del alumnado hacia Didáctica General y los enfoques de aprendizaje, predominando un enfoque profundo (pretest) y en el postest, enfoque superficial. Groves (2005, citado en González- García et al., 2019) afirma que el uso de metodologías que favorezcan el enfoque profundo puede propiciar un enfoque superficial debido a la carga de trabajo y al estilo de evaluación.

\section{OBJETIVO DEL ESTUDIO}

El objetivo general es analizar la relación entre metodologías docentes (profesorado de primer y tercer curso) y enfoques de aprendizaje utilizadas por el alumnado.

\section{MUESTRA Y PARTICIPANTES}

La muestra utilizada son dos grupos- clase naturales de $1^{0}$ y 3 er Grado de Pedagogía de la Facultad de Ciencias de la Educación de Universidad de Granada y dos profesores de la misma facultad. En la Tabla 1 se observa de primero han participado 26 estudiantes (mujeres) y de tercero han participado 35 estudiantes (5 hombres y 30 mujeres).

Tabla 1

Frecuencia según el grupo-clase

\begin{tabular}{lccc}
\hline Grupo & Frecuencia & Hombre & Mujer \\
\hline $1^{\text {o }}$ & 26 & - & 26 \\
$3^{\text {o }}$ & 35 & 5 & 30 \\
Total & 61 & 5 & 56 \\
\hline
\end{tabular}

Se ha realizado un muestreo por conglomerados o grupos no aleatorizados, se utiliza cuando los individuos de la población a estudiar se constituyen como grupos naturales o conglomerados. En esta muestra total hay 63 sujetos (Fernández y Vallejo, 2006).

\section{METODOLOGÍA E INSTRUMENTOS UTILIZADOS}

El procedimiento seguido en este estudio fue secuencial temporalizado (Tabla 2). Para los análisis se ha utilizado el programa estadístico SPSS (Statistical Package for the Social Sciences) en su versión 21.0. Se ha llevado a cabo un análisis de estadísticos descriptivos (media y desviación típica), y un análisis de T de Student para comparar las medias y su nivel de significación. Y se ha realizado un análisis de correlaciones entre las variables de los cuestionarios I.H.E. y R-SPQ-2F.

Tabla 2

Procedimiento seguido

\begin{tabular}{lll}
\hline $\begin{array}{l}\text { Octubre/18 - } \\
\text { Noviembre/18 }\end{array}$ & Diciembre/18 - Febrero/19 & Marzo/19 - Junio/19 \\
\hline & Pasación de instrumentos: & \\
$\begin{array}{l}\text { Revisión de la } \\
\text { bibliografía }\end{array}$ & -Estudiantes (I.H.E. y R-SPQ- & $\begin{array}{l}\text { Análisis de datos } \\
\text { Elaboración de la memoria }\end{array}$ \\
& 2F) & \\
\hline
\end{tabular}


Los instrumentos utilizados son: Revised two- Factor Study Process Questionnaire (R-SPQ-2F) de Biggs et al. (2001) traducido, adaptado y validado con una muestra española por Justicia, Pichardo, Cano, Berbén y De la Fuente (en prensa). Mide los enfoques de aprendizaje (profundo y superficial). Consta de 20 ítems subdivididos en cuatro subescalas con cinco ítems cada una. El cuestionario es una escala Likert de 5 respuestas desde 'nada de acuerdo' hasta 'muy de acuerdo'. Con un análisis factorial confirmatorio y un alpha de Cronbach (GarcíaBerbén, 2008) se demostró que validez y fiabilidad son aceptables; Inventario de hábitos de estudio (I.H.E) de Fernández (2014) mide hábitos de estudio. Es multifactorial y se divide en cuatro escalas: Condiciones ambientales del estudio (.956), Planificación del estudio (.959), Utilización de materiales (.956) y Asimilación de contenidos (.955); Cuestionario de Evaluación de las Variables 'Moduladoras' del Estilo de Enseñanza en Educación Superior (CEMEDEPU) de Gargallo, Fernández y Jiménez (2007) pretende corroborar si los modelos docentes universitarios se adaptan a los requisitos del EEES, consta de 51 ítems divididos en tres escalas: Modelo Centrado en la Enseñanza (.879), Modelo Centrado en el Aprendizaje (.832) y Modelo Centrado en las Habilidades del Profesor Eficaz (.839) (Pascual, 2015).El cuestionario es una escala Likert de 5 respuestas desde 'muy en desacuerdo' hasta 'muy de acuerdo'. Fue validado con una muestra de 332 (profesorado español), con nivel de confianza (95\%).

\section{RESULTADOS ALCANZADOS}

Los resultados obtenidos se desglosan a continuación:

\section{Estudios recientes. Enfoques y estrategias de aprendizaje.}

Los resultados obtenidos, Tabla 3, muestran que los estadísticos descriptivos del I.H.E.: condiciones ambientales del estudio; planificación del estudio; utilización de materiales; asimilación de contenidos; y sinceridad; del $R-S P Q-2 F$ : enfoque profundo, superficial; motivación profunda, superficial; estrategia profunda, superficial; y del CEMEDEPU: modelo centrado en la enseñanza; modelo centrado en el aprendizaje; y las habilidades docentes del profesor eficaz. 
Tabla 3

Prueba T. Estadísticos de grupo y prueba de muestras independientes

\begin{tabular}{|c|c|c|c|c|c|c|}
\hline & Grupo & $\mathbf{N}$ & Media & $\begin{array}{l}\text { Desviación } \\
\text { estándar }\end{array}$ & $\mathbf{T}$ & $\mathbf{p}$ \\
\hline \multirow{2}{*}{$\begin{array}{l}\text { Condiciones ambientales } \\
\text { del estudio }\end{array}$} & $1^{\mathrm{o}}$ & 26 & 7.73 & 1.485 & \multirow{2}{*}{2.453} & \multirow{2}{*}{.017} \\
\hline & $3^{\circ}$ & 29 & 6.59 & 1.918 & & \\
\hline \multirow{2}{*}{ Planificación del estudio } & $1^{\circ}$ & 26 & 4.88 & 2.762 & \multirow{2}{*}{-1.10} & \multirow{2}{*}{.275} \\
\hline & $3^{\circ}$ & 29 & 5.62 & 2.178 & & \\
\hline \multirow{2}{*}{ Utilización de materiales } & $1^{\circ}$ & 26 & 8.46 & 1.067 & \multirow{2}{*}{1.255} & \multirow{2}{*}{.215} \\
\hline & $3^{\circ}$ & 29 & 8.10 & 1.047 & & \\
\hline \multirow{2}{*}{$\begin{array}{l}\text { Asimilación } \\
\text { contenidos }\end{array}$} & $1^{\circ}$ & 26 & 6.85 & 1.804 & \multirow{2}{*}{2.151} & \multirow{2}{*}{.036} \\
\hline & $3^{\circ}$ & 29 & 5.79 & 1.820 & & \\
\hline \multirow{2}{*}{ Sinceridad } & $1^{\mathrm{o}}$ & 25 & 3.84 & 2.014 & \multirow{2}{*}{-.756} & \multirow{2}{*}{.454} \\
\hline & $3^{\circ}$ & 28 & 4.21 & 1.524 & & \\
\hline \multirow{2}{*}{ Enfoque profundo } & $1^{\circ}$ & 26 & 35.15 & 6.214 & \multirow{2}{*}{1.570} & \multirow{2}{*}{.122} \\
\hline & $3^{\circ}$ & 35 & 33.06 & 4.214 & & \\
\hline \multirow{2}{*}{ Enfoque superficial } & $1^{\circ}$ & 26 & 23.88 & 6.289 & \multirow{2}{*}{-.001} & \multirow{2}{*}{-.001} \\
\hline & $3^{\circ}$ & 35 & 23.89 & 6.511 & & \\
\hline \multirow{2}{*}{ Motivacion profunda } & $1^{\circ}$ & 26 & 17.42 & 4.429 & \multirow{2}{*}{.885} & \multirow{2}{*}{.382} \\
\hline & $3^{\circ}$ & 35 & 16.57 & 2.453 & & \\
\hline \multirow{2}{*}{ Estrategia profunda } & $1^{\circ}$ & 26 & 17.12 & 3.670 & \multirow{2}{*}{.870} & \multirow{2}{*}{.390} \\
\hline & $3^{\circ}$ & 35 & 16.40 & 2.354 & & \\
\hline \multirow{2}{*}{ Motivacion superficial } & $1^{\circ}$ & 26 & 9.88 & 3.398 & \multirow{2}{*}{-.739} & \multirow{2}{*}{.463} \\
\hline & $3^{\circ}$ & 35 & 10.51 & 3.212 & & \\
\hline \multirow{2}{*}{ Estrategia superficial } & $1^{\circ}$ & 26 & 14.00 & 3.919 & 808 & 423 \\
\hline & $3^{\circ}$ & 35 & 13.26 & 3.257 & .808 & $.4 \angle 3$ \\
\hline Modelo centrado en la & $1^{\circ}$ & 1 & 29.00 & 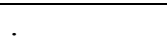 & 7444 & 085 \\
\hline enseñanza & $3^{\circ}$ & 1 & 38.00 & & 1.444 & .085 \\
\hline Modelo centrado en el & $1^{\circ}$ & 1 & 58.00 & & 5296 & 119 \\
\hline aprendizaje & $3^{\circ}$ & 1 & 85.00 & & 5.290 & .119 \\
\hline Las habilidades docentes & $1^{\circ}$ & 1 & 72.00 & & 10.000 & .063 \\
\hline del profesor eficaz & $3^{\circ}$ & 1 & 88.00 & & & \\
\hline
\end{tabular}

En relación al nivel de significación, las condiciones ambientales del estudio ( $\mathrm{t}=2.453 ; p=0.17)$ y la asimilación de contenidos ( $\mathrm{t}=2.151 ; p=0.36$ ) son significativas, con una media mayor en el alumnado de primer curso. En cambio no son significativas las demás variables: planificación del estudio (Media $1^{0}=4.88$; Media $3^{0}=5.62$ ), utilización de materiales (Media $1^{0}=8.46$; Media $3^{0}=8.10$ ), enfoque profundo (Media $1^{0}=35.15$; Media $3^{0}=$ 33.06), enfoque superficial (Media $1^{0}=23.88$; Media $3^{0}=23.89$ ), motivación profunda (Media $1^{0}=17.42$; Media $3^{0}=16.57$ ), estrategia profunda (Media $1^{0}=17.12$; Media $3^{\circ}=16.40$ ), motivación superficial (Media $1^{\circ}=9.88$; Media $3^{0}=10.51$ ) y estrategia superficial ( Media $1^{0}=14.00$; Media $3^{0}=13.26$ ).

En cuanto a los modelos docentes es mayor el centrado en la enseñanza en los estudiantes de tercero (Media $=38.00$ ), el centrado en el aprendizaje (Media $=85.00)$ y las habilidades docentes del profesor eficaz (Media = 88.00), no siendo las puntuaciones significativas en ninguna de las variables. 


\section{Correlación de Pearson y Significación bilateral.}

Se han realizado correlaciones bivariadas de los cuestionarios destinados al alumnado (Tabla 4), eliminando la variable sinceridad por su poca relación con el contenido de las demás variables.

Tabla 4

Correlaciones de Pearson de los cuestionarios I.H.E y R-SPQ-2F

\begin{tabular}{llllll}
\hline & & $\begin{array}{l}\text { Condiciones } \\
\text { ambientales del } \\
\text { estudio }\end{array}$ & $\begin{array}{l}\text { Planificación } \\
\text { del estudio }\end{array}$ & $\begin{array}{l}\text { Utilización } \\
\text { de } \\
\text { materiales }\end{array}$ & $\begin{array}{l}\text { Asimilación } \\
\text { de contenidos }\end{array}$ \\
\hline \multirow{2}{*}{ Enfoque profundo } & $\mathrm{r}$ & $.302^{*}$ & $.268^{*}$ & .198 & $.291^{*}$ \\
& $\mathrm{p}$ & .025 & .048 & .148 & .031 \\
\hline \multirow{2}{*}{ Enfoque superficial } & $\mathrm{r}$ & -.125 & -.221 & -.017 & -.205 \\
& $\mathrm{p}$ & .364 & .104 & .902 & .134 \\
\hline \multirow{2}{*}{ Motivacion profunda } & $\mathrm{r}$ & .246 & .190 & .056 & .232 \\
& $\mathrm{p}$ & .070 & .164 & .685 & .088 \\
\hline \multirow{2}{*}{ Estrategia profunda } & $\mathrm{r}$ & $.303^{*}$ & $.387^{* *}$ & .199 & .211 \\
& $\mathrm{p}$ & .024 & .003 & .145 & .122 \\
\hline \multirow{2}{*}{ Motivacion superficial } & $\mathrm{r}$ & -.149 & -.141 & .000 & -.143 \\
\hline \multirow{2}{*}{ Estrategia superficial } & $\mathrm{p}$ & .279 & .306 & .997 & .297 \\
\hline & $\mathrm{p}$ & .789 & -.143 & .022 & -.074 \\
\hline
\end{tabular}

Nota: **. La correlación es significativa al nivel 0.01 (bilateral); *. La correlación es significativa al nivel 0.05 (bilateral).

La escala de condiciones ambientales del estudio del I.H.E. tiene relación con las escalas del R-SPQ-2F: relación directa y significativa con enfoque profundo $(r=.302)$ y estrategia profunda $(r=.303)$; y relación inversa con enfoque superficial $(r=-.125)$ y motivación superficial $(r=-.149)$. La escala de planificación del estudio tiene con las escalas del R-SPQ-2F: relación directa y significativa con enfoque profundo $(r=.268)$ y estrategia profunda $(r=.387)$; y relación inversa con enfoque superficial $(r=-.221)$, motivación superficial $(r=-.141)$ y estrategia superficial $(r=-.143)$. La escala de utilización de materiales no tiene relación directa y significativa, pero tiene una relación inversa con la escala del R-SPQ-2F: enfoque superficial $(r=-.017)$. La escala de asimilación de contenidos tiene relación con las escalas del R-SPQ-2F: relación directa y significativa con enfoque profundo $(r=.291)$; y relación inversa con enfoque superficial $(r=-.205)$, motivación superficial $(r=-.143)$ y estrategia superficial $(r=-.074)$.

\section{DISCUSIÓN Y CONCLUSIONES}

Los resultados obtenidos siguen la línea de las conclusiones aportadas por diversos autores (Cruz- Tomé, 2003; Fernández de Haro, 2017; Koivuniemi et al., 2017; Pascual, 2015; Pintrich, 2000; Valerio et al., 2016; Zimmerman, 2000), la metodología docente centrada en el aprendizaje influye directamente en el enfoque, motivación y estrategia profunda utilizada por el alumnado en la Facultad de Ciencias de la Educación de Granada. Existiendo una relación directa y significativa del enfoque profundo con las escalas de condiciones ambientales del estudio, planificación del estudio y asimilación de contenidos. Por tanto, existe relación entre una adecuada metodología docente centrada en el aprendizaje y el uso de estrategias y enfoques de aprendizaje adecuados. La concepción de los procesos de E-A en el ámbito analizado demuestra que existe una posible adaptación a los nuevos retos del EEES, la función docente en las asignaturas analizadas se declina por un aprendizaje significativo por parte del alumnado confiriéndole la capacidad de seleccionar, comprender y adquirir aquella información 


\section{RELACIÓN ENTRE METODOLOGÍAS DOCENTES Y ENFOQUES DE APRENDIZAJE EN LA UNIVERSIDAD}

esencial, usando adecuadamente las estrategias de aprendizaje que le permitan obtener de forma satisfactoria las metas académicas propuestas.

Pintrich y DeGroot (1990) hallaron correlaciones positivas entre las estrategias de aprendizaje utilizadas por el alumnado y su motivación. Estudios como Rinaudo et al. (2003) reafirman la idea de que el aprendizaje está condicionado por la motivación y el uso adecuado de estrategias de aprendizaje. Alarcón et al.(2019) resalta este uso adecuado para obtener un aprendizaje autónomo y autorregulado. González- García et al. (2019) señalan que el alumnado puede desarrollar un enfoque profundo, y posteriormente tornar hacia superficial. González, Del Rincón y Del Rincón (2011) subrayaban que los enfoques no son 'estáticos', dependen del contexto.

Los hallazgos obtenidos se observan mayores puntuaciones (enfoque profundo) en primero que en tercero, puede deberse a factores no controlados (percepción por parte del alumnado de la utilidad de la asignatura en su futuro profesional; la asignatura con matiz más teórico o práctico; la asignatura de primero es básica y la de cuarto es optativa, ...). Las limitaciones de este estudio son: la muestra que se ha utilizado es pequeña (63 participantes) lo cual hace difícil la generalización de resultados, pero nos capacita para tener una visión unitaria de los cambios que se están dando en nuestro contexto. Un posible estudio futuro sería de carácter longitudinal que implique más asignaturas, más facultades que pueda aportar resultados adicionales que nos permitan ver la realidad actual desde otra perspectiva, modulando las variables con el fin de obtener resultados más significativos y extrapolables al resto de la población.

\section{REFERENCIAS BIBLIOGRÁFICAS}

Alarcón, A., Alcas, N., Alarcón, H., Natividad, J., y Rodríguez, A. (2019). Empleo de las estrategias de aprendizaje en la universidad. Un estudio de caso. Propósitos y Representaciones, 7(1). doi: 10.20511/pyr2019.v7n1.265

Biggs, J. (1987). Student approaches to learning and studying. Hawthorn: Australian Council for Educational Research.

Biggs, J. (1988). The role of metacognition in Enhancing learning. Australian Journal of Education, 32, 127-138. doi: $10.1177 / 000494418803200201$

Biggs, J. (1989). Approaches to the enhancement of tertiary teaching. Higher Education Research and Development, 8(1), 7-25.doi: 10.1080/0729436890080102

Biggs, J. (1993). What do inventories of students' learning processes really measure? A theoretical review and clarification. British Journal of Educational Psychology, 63, 3-19. doi: 10.1111/j.2044-8279.1993.tb01038.x

Biggs, J. (1999). Teaching for Quality Learning at University. Buckingham: Open University Press.

Biggs, J. (2001). Enhancing learning: a matter of style or approach? En R.J. Sternberg, y L-F. Zhang, Perspectives on Thinking, Learning and Cognitive Style (pp.73-102). London: LEA.

Biggs, J., Kember, D., y Leung, D. (2001). The Revised two- factor Study Process Questionnaire: R-SPQ-2F. British Journal of Educational Psychology, 71, 133-149. doi: 10.1348/000709901158433

Cano, F. (2007). Tema 18: Aprendizaje estratégico. En E. Fernández de Haro, F. Justicia, y M. C. Pichardo, Enciclopedia de Psicología Evolutiva y de la Educación. Vol. 1. (pp.485-508). Málaga: Aljibe.

Cruz-Tomé, M.A. (2003). El proceso de convergencia europea: ocasión para modernizar la universidad española si se produce un cambio de mentalidad en gestores, profesores y estudiantes. Aula Abierta, 82, 191-216. Recuperado de: https://dialnet.unirioja.es/servlet/articulo?codigo=1012087

Entwistle, N.J. (1988). Motivational factors in students' approaches to learning. En R.R. Schmeck (ed.), Learning Strategies and Learning Styles (pp.21-50). New York: Plenum Press.

Fernández de Haro, E. (2017). ¿Por qué son importantes las técnicas de estudio en la universidad? ¿Cuáles me ayudan a aprender?. En J. A. Pareja Fdez de la Reguera (coord), Manual para la adquisición de algunas de las competencias transversales en el grado de pedagogía (pp.49-70).

Fernández, A., y Vallejo, M. (2006). Evaluación de programas, centros y profesores. Cuaderno de metodología. Granada: Grupo Editorial Universitario. 
Fernández, F. (2014). IHE. Inventario de hábitos de estudio. Ediciones TEA.

García-Berbén, A.B. (2008). Proceso de enseñanza/aprendizaje en Educación Superior. (Tesis doctoral). Universidad de Granada. Recuperado de: http://hdl.handle.net/10481/1976

Gargallo, B., Fernández, A., y Jiménez, M.A. (2007). Modelos docentes de los profesores universitarios, Teoría de la educación, 19, 167-189. doi: http://dx.doi.org/10.14201/teri.3256

González, J.L., del Rincón, B., y del Rincón, D.A. (2011). Estructura latente y Consistencia interna del R-SPQ-2F: Reinterpretando los enfoques de aprendizaje en el EEES. Revista de Investigación Educativa, 29 (2), 277-293. Recuperado de: https://revistas.um.es/rie/article/view/112431

González-García, N., Sánchez-García, A.B., Nieto-Librero, A.B., y Galindo-Villardón, M. P. (2019). Attitude and Learning Approaches in the Study of General Didactics. A Multivariate Analysis. Revista de Psicodidáctica (English ed.), 24(2), 154-162. doi: 10.1016/j.psicoe.2019.03.001

Justicia, F., Pichardo, M.C., Cano, F., García-Berbén, A.B., y De la Fuente, J. (en prensa). The Revised Two- Factor Study Process Questionnaire (R-SPQ-2F): Exploratory and Confirmatory Factor Analyses at ítem Level. European Journal of Psychology of Education, XXIII.

Koivuniemi, M., Panadero, E., Malmberg, J., y Järvelä, S. (2017). Higher education students' learning challenges and regulatory skills in different learning situations / Desafíos de aprendizaje y habilidades de regulación en distintas situaciones de aprendizaje en estudiantes de educación superior, Infancia y Aprendizaje, 40(1), 1955. doi: 10.1080/02103702.2016.1272874

Martínez, J.R., y Galán, F. (2000). Estrategias de aprendizaje, motivación y rendimiento académico en alumnos universitarios, REOP, 11(19), 35-50. doi: 10.5944/reop.vol.11.num.19.2000.11323

Marton, F. (1975). On Non- Verbatim Learning I: Level of Processing and Level of Outcomes, Scandinavian Journal of Psychology, 16, 273-279. doi: 10.1111/j.1467-9450.1975.tb00193.x

Marton, F., y Säljö, R. (1976a). On qualitative Differences in learning I-Outcome and Process. British Journal of Psychology, 46, 4-11. doi: 10.1111/j.2044-8279.1976.tb02980.x

Marton, F., y Säljö, R. (1976b). On qualitative Differences in learning II-Outcome and Process. British Journal of Psychology, 46, 115-127. doi: 10.1111/j.2044-8279.1976.tb02304.x

Marton, F., y Säljö, R. (1984). Approaches to learning. En F. Marton, D.J. Hounsell y N.J. Entwistle (Eds.), The experience of learning. Edinburgh: Scottish Academic Press.

Marton, F., y Svensson, L. (1979). Conceptions of research in Student eLearning. Higher Education, 8, 471-486. doi: 10.1007/BF01680537

Pascual, H.M. (2015). Análisis de la influencia del espacio europeo de educación superior (EEES) en el cambio y la motivación del alumnado universitario y su profesorado en la titulación de magisterio. Estudio comparado con el Reino Unido. (Tesis doctoral). Universidad de Granada. Recuperado de: http://hdl.handle.net/10481/42259

Pintrich, P. (2000). The role of goal orientation in self-regulated learning. En M. Boekaerts, P. Pintrich, y M. Zeidner (Eds.), Handbook of self-regulation (451-502). San Diego, CA: Academic Press.

Pintrich, P., y DeGroot, E. (1990). Motivational and self-regulated learning components of classroom Academic performance, Journal of Educational Psychology, 82(1), 33-40. Doi: 10.1037/0022-0663.82.1.33

Prosser, M., y Trigwell, K. (1999). Understanding Learning and Teaching. Buckingham: Open University Press.

Ramsden, P. (2003). Learning to teach in Higher education (2 Ed.). New York: RoutledgeFalmer.

Rinaudo, M.C., Chiecher, A., y Donolo, D. (2003). Motivación y uso de estrategias en estudiantes universitarios. Su evaluación a partir del Motivated Strategies Learning Questionnaire. Anales de Psicología, 19(1), 107119. Recuperado de: https://www.redalyc.org/articulo.oa?id=16701911

Romero, A.J., Jordan, G., Ilaquiche, R., García, G., y Tapia, H.P. (2019). Percepciones de los docentes sobre el uso de los estilos de aprendizaje en el desarrollo de competencias de aprender a pensar y pensar para aprender. Revista Espacios, 40 (5), 23. Recuperado de: https://revistaespacios.com/a19v40n05/19400523.html 


\section{RELACIÓN ENTRE METODOLOGíAS DOCENTES Y ENFOQUES DE APRENDIZAJE EN LA UNIVERSIDAD}

Valerio, G., Jaramillo, J., Caraza, R., y Rodríguez, R. (2016). Principios de Neurociencia aplicados en la Educación Universitaria. Formación Universitaria, 9 (4), 75-82. doi: 10.4067/S0718-50062016000400009

Valle, A., Cabanach, R.G., y Vieiro, P. (1997). Características diferenciales de los enfoques de aprendizaje en estudiantes universitarios. Revista de Psicodidáctica, 4, 41-58. Recuperado de: https://www.redalyc.org/articulo.oa?id=17517797003

Vermunt, J.D. (2003). The power of learning environments and the Quality so Student learning. En E. De Corte, L. Verschaffel, N. Entwistle y J. Van Merriënboer (Eds.), Powerful learning Environments: Unravelling Basic Components and Dimensions. London: Pergamon y Earli.

Weinstein, C.E., y Mayer, R.E. (1986). The teaching of learning strategies. En Wittrock, M.C. (Ed.), Handbook of research on teaching. (pp. 315-327). New York: Macmillan.

Zimmerman, B. (2000). Attaining self-regulation. A social cognitive perspective. En M. Boekaerts, P. Pintrich, y M. Zeidner (Eds.), Handbook of self-regulation (pp. 13-39). San Diego: Academic Press.

\section{FINANCIACIÓN}

Este estudio está financiado por una Beca de Iniciación a la Investigación para Estudiantes de Másteres Oficiales del Plan Propio de Investigación y Transferencia 2018 otorgada a Marta García Jiménez con N.I.F. №76626510D bajo la dirección de la profesora María Fernández Cabezas en el Departamento de Psicología Evolutiva y de la Educación de la Universidad de Granada. 